\title{
FOMENTO DE LA LECTURA A TRAVÉS DE LAS TIC
}

Promoting reading through ITC

Promoção da leitura através das TIC

\author{
Pablo Santín Álvarez (1) \\ Zaira Bada Fernández (2)
}

(1) CEIP Monte Gándara, El Puente, Zamora. Teléfono: +34 980620233. Correo

electrónico: pablo.sanalv.3@educa.jcyl.es

(2) IES Guardo, Guardo, Palencia. Teléfono: +34 979852298. Correo electrónico:

\section{zaira.badfer@educa.jcyl.es}

\begin{abstract}
Resumen
Esta comunicación describe una experiencia educativa sobre el fomento de la lectura a través de la realización de un video-tutorial. En este video-tutorial cada alumno hace una pequeña reseña y recomendación sobre un libro escogido en la biblioteca del centro, el cual previamente haleído. Para su elaboración, el alumnado cuenta con una escala graduada en la cual se especifica los criterios de calificación. Dicha experiencia tiene una triple funcionalidad puesto que a partir de su puesta en práctica se está trabajando con ella expresión oral, la competencia digital a través de la utilización de las tecnologías de la información y comunicación (TIC) y además ha aumentado considerablemente el interés por la lectura.

Palabras clave: Lectura; video-tutorial; biblioteca; TIC
\end{abstract}

\begin{abstract}
This communication describes an educational experience on the promotion of reading through the realization of a video tutorial. In this video tutorial each student makes a small review and recommendation about a book chosen in the library of the center, which has previously read. For its elaboration, the students have a graduated scale in which the qualification criteria are specified. This experience has a triple functionality that comes from its implementation with oral expression, digital competition through
\end{abstract}


the use of information and communication technologies (ICT) and also has greatly increased interest in reading.

Keywords: Reading; video-tutorial; library; ICT

\section{Resumo}

Este artigo descreve uma experiência educativa sobre promoção da leitura através de fazer um vídeo tutorial. Neste vídeo tutorial cada aluno faz uma breve revisão e recomendação sobre um livro escolhido na biblioteca da escola, que leu anteriormente. Para o processamento, o aluno tem uma escala em que os critérios de qualificação são especificados. Esta experiência tem uma funcionalidade triplo em sua implementação com a expressão oral, competência digital através do uso de tecnologia da informação e comunicações (TIC) e também aumentou muito o interesse pela leitura.

Palavras-chave: Leitura; vídeotutorial; biblioteca; TIC

\section{Introducción}

Esta comunicación analiza una experiencia de animación a la lectura a través de la utilización de las tecnologías de la información y comunicación (TIC), aprovechando todo los recursos de los que se disponen en el centro y dando especial protagonismo tanto a la biblioteca de aula como a la del centro.

La principal función de esta experiencia es desarrollar el gusto por la lectura en el alumnado cumpliendo así con uno de los objetivos que establece elReal Decreto $126 / 2014$.

Lo que se trata es de formar alumnos con criterio propio, que sean capaces de distinguir entre aquello que les gusta y lo que no,ofreciéndoles todas las posibilidades disponibles a su alcance (Mata, 2009).Además, a través de la utilización de las diferentes herramientas TIC se pretende mostrar al alumnado las posibilidades que ofrecen y lo que es más importante, se está procurando que nuestros alumnos sepan manejar estas herramientas, es decir, que sean competentes en este campo, lo cual recoge la orden ECD/65/2015.

En definitiva, se persigue que el alumnado a través del desarrollo del hábito lector y la utilización de diferentes herramientas TIC pueda llegar a construir su propio 
aprendizaje convirtiéndose así en generadores de su propio conocimiento (Lozano, 2011).

\section{Contextualización}

La experiencia se lleva a cabo en un colegio público de línea 1 de un entorno rural de la provincia de Zamora. La etapa educativa en la que se ha desarrollado es Educación Primaria, concretamente en una clase de $5^{\circ}$ curso que cuenta con 13 alumnos. Se trata de un grupo heterogéneo, algunos de ellos con necesidades específicas de apoyo educativo, los cuales también han realizado la actividad.

\section{Diseño y desarrollo}

Para llevar a cabo la experiencia se les pidió a los alumnos que escogieran uno de los libros de la biblioteca del centro para posteriormente leerlo. Una vez leído, cada alumno elaboró un video-tutorial en el que presentaron el libro, los personajes, etc. Se trata de elaborar una pequeña sinopsis con el objetivo de recomendar ese libro al resto de sus compañeros. Para ello, previamente, se les había entregado una escala graduada (Tabla 1), modificada de Pérez-Pueyo y Casado (2015) en la que pueden encontrar todos los aspectos que se van a valorar y los niveles de logro.

En un primer momento, la iniciativa era exclusivamente para el grupo-clase, pero se extendió a la biblioteca del colegio a través de la colocación de un código QR en cada uno de los libros. Para ello, cada alumno asocia la dirección de su video-tutorial a un código QR a través de la página QR CodeGenerator. El código generado se pega en la contraportada del libro. Esto posibilita que cada alumno pueda acudir a la zona de la biblioteca donde se encuentra la estantería de los libros recomendados y comprobar por qué lo recomiendan sus compañeros accediendo al video-tutorial con la aplicación QR DroidCode Scanner.

Por otro lado, destacar que todos estos video-tutoriales se suben al canal de Youtube del colegio, por lo tanto se convierten en una plataforma para que los alumnos de otros colegios puedan acudir a ella a la hora de escoger un libro de lectura.

\section{Evaluación y conclusiones}

Para la evaluación de la actividad, como es obvio se utilizará la misma escala graduada que se les entrega a los alumnos antes de la realización del video-tutorial (Tabla 1). Antes de la entrega final, los alumnos tienen dos fechas de entrega anteriores a esta. Por 
lo tanto se está llevando a cabo una evaluación formativa, ya que se les da la oportunidad de mejorar su trabajo (López-Pastor \& Pérez Pueyo, 2017).

Tabla 1.

Escala grachada del video-tutorial adaptada de Pérez-Pueyo y Casado (2015).

\begin{tabular}{|c|c|}
\hline \multirow{6}{*}{ 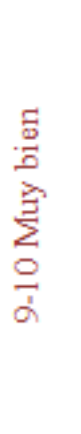 } & $\begin{array}{l}\text { Presentanel conterido solicitado: Título del libro y autor, presentación de los personajes, } \\
\text { breve resumen del libro, recomendación y despedida. }\end{array}$ \\
\hline & Se presentantodos los aspectos a identificar como el nombre del alumno, centro o curso. \\
\hline & La duración no excede de $2 \mathrm{~min}$. \\
\hline & $\begin{array}{l}\text { Se observa una lógica en el contado y se destacan/resaltan los aspectos importantes a } \\
\text { tener en cuenta. }\end{array}$ \\
\hline & Se sigue el guion preestablecido. \\
\hline & La calidad de la grabación y el cuidado del vídeo son destacables. \\
\hline \multirow{6}{*}{ 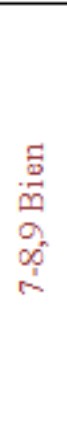 } & $\begin{array}{l}\text { Presentanel conterido solicitado de manera casi completa (Por ejemplo, se tratan todos } \\
\text { los apartados de un tema aunque en algunos casos de manera superficial). }\end{array}$ \\
\hline & Falta algún elemento por identificar como nombre, curso o colegio. \\
\hline & La duración no excede de $2 \mathrm{~min}$, pero hay partes que sobran. \\
\hline & $\begin{array}{l}\text { En algunos momentos se pierde la lógica en el contado y no se destacan/resaltan aspectos } \\
\text { importantes a tener en cuenta. }\end{array}$ \\
\hline & Se presentó guion pero no se transformó/reflejó en el resultado. \\
\hline & La calidad de la grabación y el cuidado del vídeo son mejorables pero suficientes. \\
\hline \multirow{6}{*}{ 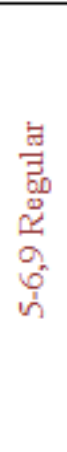 } & $\begin{array}{l}\text { Presentan el conterido de manera incompleta (Por ejemplo, algunos apartados del tema } \\
\text { no se tratan). }\end{array}$ \\
\hline & $\begin{array}{l}\text { No se presentan varios de los aspectos a identificar como nombre del alumno, centro o } \\
\text { curso. }\end{array}$ \\
\hline & La duración no excede de $2 \mathrm{~min}$ pero no de $3 \mathrm{~min}$ y hay cosas que sobran. \\
\hline & $\begin{array}{l}\text { No se observa una lógica ni en el contado ni se destacan/resaltan la mayoría } \\
\text { aspectos importantes a tener en cuenta. }\end{array}$ \\
\hline & No se presentó el guion. \\
\hline & La calidad de la grabacióny el cuida do del vídeo se deberían haber trabajado mucho más. \\
\hline \multirow{3}{*}{$\begin{array}{l}\text { J } \\
\sum_{0} \\
\dot{0} \\
0\end{array}$} & No se presentan el contenido mínimo o la información básica. \\
\hline & La duración supera los $3 \mathrm{~min}$. \\
\hline & No hay explicaciones ni/o comentarios explicativos. \\
\hline
\end{tabular}

Con la puesta en práctica de esta experiencia ha aumentado la lectura de libros de forma voluntaria por parte de los alumnos. Además, el interés por la biblioteca se ha incrementado notablemente, lo cual provoca que el alumnado no vea este espacio como una zona de castigo o de visita obligada; por lo se puede decir que se ha dado un paso adelante hacia la creación del hábito de lectura. 
El hecho de que sea un compañero el que te recomienda la lectura del libro aumenta las posibilidades de que otro alumno quiera leer dicho libro ya que entre ellos conectan con sus intereses y sus gustos (Rodríguez, Fernández \& Escudero, 2002). De esta forma son ellos mismos los que están fomentando el hábito lector entre sus compañeros.

A través de la utilización de estas herramientas se está trabajando la competencia digital, además de la expresión oral de nuestros alumnos; aspectos fundamentales en la actualidad.

Por último, destacar que, aunque comenzó como una experiencia destinada al grupo-aula, se ha extendido en un primer momento a la biblioteca del centro y en un segundo momento a otros colegios con los que se está en colaboración; por tanto, es un claro indicativo de que está práctica funciona y consigue que los alumnos se conviertan en lectores activos y con criterio.

\section{Referencias}

López-Pastor, V. M., \& Pérez-Pueyo, Á. (cood.) (2017). Evaluación formativa y compartida en educación: experiencias de éxito en todas las etapas educativas. León: Universidad de León. Recuperado de http://buleria.unileon.es/handle/10612/5999

Lozano, R. (2011). De las TIC a las TAC: tecnologías del aprendizaje y el conocimiento. Anuario ThinkEPI, 5, 45-47.

Mata, J. (2009). 10 ideas clave. Animación a la lectura: Hacer de la lectura una pràctica feliz, trascendente y deseable. Barcelona: Graó.

Orden ECD/65/2015, de 21 de enero, por la que se describen las relaciones entre las competencias, los contenidos y los criterios de evaluación de la educación primaria, la educación secundaria obligatoria y el bachillerato.

Pérez-Pueyo, A., \& Casado, O. M., (2015). Escala graduada para la valoración de un video-tutorial [documento de descarga]. Recuperado de http://www.grupoactitudes.com/\#!competencias-bsicas-nueva/c1dub

Real Decreto 126/2014, de 28 de febrero, por el que se establece el currículo básico de la Educación Primaria. 
Rodríguez, L. M., Fernández, R., \& Escudero, T. (2002). Aprendizaje entre iguales y construcción de conceptos. Infancia y aprendizaje, 25(3), 277-297. 